\title{
The relationship between nerve conduction studies and neuropathic pain in sciatic nerve injury due to intramuscular injection
}

\author{
Halit Fidancı $^{1,2}$ and İlker Öztürk ${ }^{2}$ \\ 'Department of Neurology, Division of Clinical Neurophysiology, Adana City Training and Research Hospital, Adana, Turkey \\ ${ }^{2}$ Department of Neurology, Adana City Training and Research Hospital, Adana, Turkey
}

Received August 24, 2020

Revised November 28, 2020

Accepted December 9, 2020

Handling Editor: Jae Hun Kim

\section{Correspondence}

Halit Fidancı

Department of Neurology, Division of Clinical Neurophysiology, Adana City Training and Research Hospital, Yüregir, Adana 01060, Turkey

Tel: +90322455900-1147

Fax: +903223440305

E-mail: dr.halitfidanci@gmail.com
Background: Sciatic nerve injury due to intramuscular injection (SNIII) is still a health problem. This study aimed to determine whether there is a correlation between neuropathic pain and electrodiagnostic findings in SNIII.

Methods: Patients whose clinical and electrodiagnostic findings were compatible with SNIII participated in this retrospective cohort study. Compound muscle action potential (CMAP) and sensory nerve action potential (SNAP) amplitudes of the sural, superficial peroneal, peroneal, and tibial nerves were graded from 1 to 4 . Leeds assessment of neuropathic symptoms and signs scale (LANSS) was applied to all patients.

Results: Forty-eight patients were included in the study, $67 \%$ of whom had a LANSS score $\geq 12$. Sural SNAP amplitude abnormalities were present in $8(50 \%)$ out of 16 patients with a LANSS score $<12$, and $28(87.5 \%)$ out of 32 patients with a LANSS score $\geq 12$, with significant differences between the groups $(P=0.011)$. There was a positive correlation between the LANSS score and the sural SNAP amplitude grading $(P=0.001, r=0.476)$. A similar positive correlation was also found in the LANSS score and the tibial nerve CMAP amplitude grading $(P=0.004, r=0.410)$.

Conclusions: This study showed a positive correlation between the severity of tibial nerve CMAP/sural SNAP amplitude abnormality and LANSS score in SNIII. Neuropathic pain may be more common in SNIII patients with sural nerve SNAP amplitude abnormality.

Key Words: Action Potentials; Electrodiagnosis; Electromyography; Injections, Intramuscular; Neural Conduction; Neuralgia; Sciatic Nerve; Sciatic Neuropathy; Sural Nerve; Tibial Nerve.

\section{INTRODUCTION}

Sciatic nerve (SN) injury is a well-known condition that can occur due to hip surgery, gluteal intramuscular (IM) injection, compression, or hip lesions [1-4]. Despite the precautions taken, SN injury due to IM injection (SNIII) still continues to be a health problem. SNIII can cause clinical manifestations ranging from mild sensory complaints to severe motor weakness [1-3]. Weakness in the muscles innervated by the SN and its branches, as well as sensory abnormalities over the skin areas supplied by these nerves, result from SN injury. Pain occurs in the ma- (c) This is an open-access article distributed under the terms of the Creative Commons Attribution Non-Commercial License (http://creativecommons.org/licenses/by-nc/4.0/), which permits unrestricted non-commercial use, distribution, and reproduction in any medium, provided the original work is properly cited.

(c) The Korean Pain Society, 2021
Author contributions: Halit Fidancl: Writing/manuscript preparation; ilker Öztürk: Investigation. 
jority of the patients, which may limit their daily activities. Allodynia or hyperalgesia may develop [1,4].

The diagnosis of SNIII can be made using clinical findings and electrodiagnostic tests. Nerve conduction studies and needle electromyography (EMG) have an important place both in the actual diagnosis and differential diagnosis of SNIII. In this study, we aimed to determine whether there is a relationship between neuropathic pain and nerve conduction studies on SNIII. Thus, we wanted to obtain information about the pathophysiology of neuropathic pain in SNIII.

\section{MATERIALS AND METHODS}

\section{Subjects}

The present study was approved by the Institutional Review Board (IRB) of Adana City Training and Research Hospital (IRB number: 45/623). All participants provided written informed consent and the study complied with the ethical principles as set out by the Declaration of Helsinki. Patients who were admitted to our EMG laboratory between July 2018 and February 2020, and whose clinical and electrodiagnostic findings were compatible with SNIII, were included in this retrospective cohort study.

Patient complaints had to occur after gluteal IM injection. The injection was administered by a paramedic or a nurse into the gluteus maximus muscle of the patients. The injection site was the outer upper quadrant of the buttock. The length of the needle used for administration for IM injection was $38 \mathrm{~mm}$. In addition, patients needed to have at least one of the following: weakness in the muscles innervated by the SN or its branches; sensory abnormality in skin areas supplied by the branches of the SN; abnormality in nerve conduction studies of the branches of the $\mathrm{SN}$; needle EMG abnormality in the muscles innervated by the SN or its branches. Individuals with one of the following characteristics were excluded from the study: polyneuropathy, a disease which may cause neuropathy, or electrodiagnostic test or magnetic resonance imaging (MRI) findings compatible with lumbosacral radiculopathy or plexopathy. Clinical, neurological examination, electrodiagnostic test, and MRI findings of the patients were analyzed.

In order to exclude lumbosacral radiculopathy, the lumbosacral paraspinal muscles of the patient needed to be examined with needle EMG and/or a lumbosacral MRI of the patient needed to be available. In patients with suspected lumbosacral plexopathy, saphenous and femoral nerve conduction studies were performed and needle EMG was applied to the vastus lateralis, gluteus maximus, and gluteus medius muscles according to the pain tolerance of the patient. Data of 54 patients were examined. Since two patients had diabetes mellitus and four patients had lumbosacral MRI or electrodiagnostic findings compatible with lumbosacral radiculopathy, these patients were excluded from the study. Finally, forty-eight patients were included.

The Turkish version of the Leeds assessment of neuropathic symptoms and signs scale (LANSS) was applied to all patients [5]. The LANSS consisted of two parts: the pain questionnaire (LANSS-A) and sensory testing (LANSS$B)$. The LANSS-A, LANSS-B, and LANSS total (LANSS-T) scores were calculated. On the LANSS scale, 12 or more points indicated neuropathic pain [5]. Therefore, patients were divided into two groups: those with a LANSS-T score $<12$ and $\geq 12$.

\section{Electrodiagnostic tests}

Nerve conduction studies and the needle EMG were performed with the Cadwell Sierra Summit EMG unit (Cadwell Laboratories, Kennewick, WA). Stimulation and recording were performed with surface electrodes in the nerve conduction studies. If the temperature of the limb was $\geq 32^{\circ} \mathrm{C}$, electrodiagnostic tests were performed. Cold extremities were heated. Electrodiagnostic tests were performed on both lower extremities and one of the upper extremities. Low-high band filters for sensory and motor nerve conduction studies were set at $20 \mathrm{~Hz}-2 \mathrm{kHz}$ and $20 \mathrm{~Hz}-10 \mathrm{kHz}$, respectively. The sweep speed and sensitivity for sensory nerve conduction studies were $1 \mathrm{~ms} /$ division and $10 \mu \mathrm{V} /$ division, respectively. The sweep speed and sensitivity for the motor nerve conduction studies were $5 \mathrm{~ms} /$ division and $2 \mathrm{mV} /$ division, respectively. The amplitudes of the sensory nerve action potential (SNAP) and compound muscle action potential (CMAP) were calculated by measuring from peak to peak.

The sural and superficial peroneal sensory nerve conduction studies were performed antidromically, and the stimulation was performed $12-14 \mathrm{~cm}$ proximal to the recording electrode. Superficial peroneal sensory nerve conduction velocity was calculated using onset latency. Sural nerve conduction velocity was calculated using peak latency. The tibial nerve CMAP was recorded from the abductor hallucis muscle and the peroneal nerve CMAP from both the extensor digitorum brevis and the tibialis anterior (TA) muscles. The tibial and peroneal nerve was stimulated $10 \mathrm{~cm}$ and $8 \mathrm{~cm}$ proximal to the recording electrodes, respectively. To exclude peroneal neuropathy at the fibular head, the peroneal nerve was stimulated at the ankle, below the fibular head, and at the popliteal fossa. Median and ulnar nerve conduction studies were performed using 


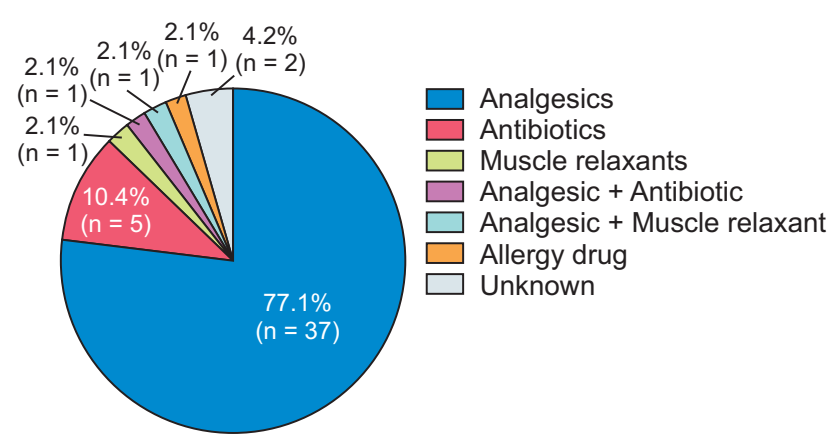

Fig. 1. Intramuscular (IM) agents associated with sciatic nerve injury.

conventional methods as mentioned in our previous study [6].

The reference values for nerve conduction studies in our previous studies were used as upper or lower limits of normal values $[6,7]$. The reference lower limits for CMAP amplitudes of the tibial nerve recorded from the abductor hallucis muscle and the peroneal nerve recorded from the extensor digitorum brevis/TA muscle were $4.2 \mathrm{mV}$ and 3.7/3.9 mV, respectively. The reference lower limit for the sural and superficial peroneal nerve SNAP amplitude was $5.1 \mu \mathrm{V}$ and $5.3 \mu \mathrm{V}$, respectively. If the CMAP or SNAP amplitude is lower than the reference lower limit, or lower than $50 \%$ of the CMAP or SNAP amplitude of the intact limb, the CMAP or SNAP amplitude was considered abnormal. The CMAP or SNAP amplitude classification was as follows [2]: Grade 1: Normal CMAP or SNAP amplitude (CMAP or SNAP amplitude that did not meet the criteria of Grade 2, 3, or 4); Grade 2: CMAP or SNAP amplitude was between $50 \%$ and $100 \%$ of the reference lower limit or between $40 \%$ and $50 \%$ of the intact side; Grade 3: CMAP or SNAP amplitude was less than $50 \%$ of the reference lower limit or less than $40 \%$ of the intact side; Grade 4: Absent CMAP or SNAP.

Needle EMG was performed visually using a concentric needle electrode (length $=50 \mathrm{~mm}$, diameter $=0.46 \mathrm{~mm}$, Bionen Medical Devices, Florence, Italy). The low-high band filter for needle EMG was $10 \mathrm{~Hz}-10 \mathrm{kHz}$. The sensitivity for active denervation and motor unit action potential (MUAP) analysis were $100 \mu \mathrm{V} /$ division and 500-1,000 $\mu \mathrm{V} /$ division, respectively, and the sweep speed was $10 \mathrm{~ms} /$ division for both analyses. The presence of positive sharp waves and fibrillation potentials were carefully examined. The MUAP analysis was performed during mild muscle contraction. If MUAP duration was $>15 \mathrm{~ms}$ and amplitude $>4 \mathrm{mV}$, the MUAP was considered neurogenic. According to the tolerance level of the patient, needle EMG was applied to the $\mathrm{TA}$, medial gastrocnemius, peroneus longus, short head of the biceps femoris, vastus lateralis, gluteus medius, gluteus maximus, as well as the L3, L4, L5, and S1 paraspinal muscles of the patient.
Table 1. Neurological examination of the patients

\begin{tabular}{lc}
\hline \multicolumn{1}{c}{ Neurological examination } & Value \\
\hline Abnormality in sensory examination & \\
Dorsum of the foot/lateral of the leg & $41(85)$ \\
Sole of the foot & $32(67)$ \\
Posterolateral leg & $30(63)$ \\
None & $2(4)$ \\
Weakness & \\
Dorsiflexion or eversion of the foot & $40(83)$ \\
Plantar flexion or inversion of the foot & $26(54)$ \\
Knee flexion & $32(67)$ \\
None & $3(6)$ \\
\hline
\end{tabular}

Values are presented as number (\%).

\section{Statistical analysis}

The Shapiro-Wilk test was used to determine the distribution of the data. The Pearson's chi-squared and Fisher's exact tests were used to analyze categorical variables. The Spearman's test was used for correlation. The mean \pm standard deviation, median, and minimum-maximum of numeric data were calculated for descriptive statistics. A $P$ value of less than 0.05 was considered significant. The Statistical Package for the Social Sciences ver. 22.0 (IBM Co., Armonk, NY) was used to perform the statistical analysis.

\section{RESULTS}

Forty-eight patients (36 males, 12 females) with SNIII were included in the study. The mean age of the patients was $41.4 \pm 15.8$ (ranging from 19 to 79 ) years. The mean body mass index of the patients was $21.8 \pm 3.4$ (ranging from 15.6 to 31.9$) \mathrm{kg} / \mathrm{m}^{2}$. The time interval between the electrodiagnostic tests and the first complaints was $9.3 \pm 9.8$ (min-max 0.7-36) months. The reason for IM injection was infection $(n=17)$, muscle-joint pain or generalized pain $(n=18)$, abdominal pain $(\mathrm{n}=5)$, headache $(\mathrm{n}=4)$, toothache $(\mathrm{n}=$ $3)$, or allergy $(n=1)$. IM agents are shown in Fig. 1. Thirtytwo patients had SN injuries in the left lower extremity. In 42 patients, complaints started within seconds of IM injection, while in six patients, complaints began within minutes to hours.

The neurological examination summary of the patients is shown in Table 1. SNAP and CMAP amplitude grading of patients are provided in Table 2 . The nerve conduction study was normal in one patient, but this patient had positive sharp waves and fibrillation potentials in the TA muscle. All patients had needle EMG abnormalities in at least one muscle innervated by the SN or its branches. Needle EMG was applied to the TA and medial gastrocnemius muscles of 48 patients, biceps femoris short head muscle 
Table 2. CMAP and SNAP amplitude grading in patients

\begin{tabular}{lccrr}
\hline \multicolumn{1}{c}{ Nerve } & ${\text { Grade } 1^{\mathrm{a}}}$ & ${\text { Grade } 2^{\mathrm{b}}}$ & ${\text { Grade } 3^{\mathrm{c}}}$ & ${\text { Grade } 4^{\mathrm{d}}}$ \\
\hline Sural & $12(25)$ & $12(25)$ & $10(21)$ & $14(29)$ \\
Superficial peroneal & $16(33)$ & $5(10)$ & $7(15)$ & $20(42)$ \\
Peroneal (EDB) & $18(38)$ & $7(15)$ & $11(23)$ & $12(25)$ \\
Peroneal (TA) & $29(60)$ & $3(6)$ & $10(21)$ & $6(13)$ \\
Tibial & $18(38)$ & $10(21)$ & $11(23)$ & $9(19)$ \\
\hline
\end{tabular}

Values are presented as number (\%).

CMAP: compound muscle action potential, SNAP: sensory nerve action potential, EDB: extensor digitorum brevis, TA: tibialis anterior.

${ }^{a}$ Normal CMAP or SNAP amplitude (CMAP or SNAP amplitude that does not meet the criteria of Grade 2, 3, or 4). ${ }^{b} \mathrm{CMAP}$ or SNAP amplitude is between $50 \%$ and $100 \%$ of the reference lower limit or $40 \%$ and $50 \%$ of the intact side. ${ }^{\circ} \mathrm{CMAP}$ or SNAP amplitude is less than $50 \%$ of the reference lower limit or less than $40 \%$ of the intact side. ${ }^{d}$ Absent CMAP or SNAP.

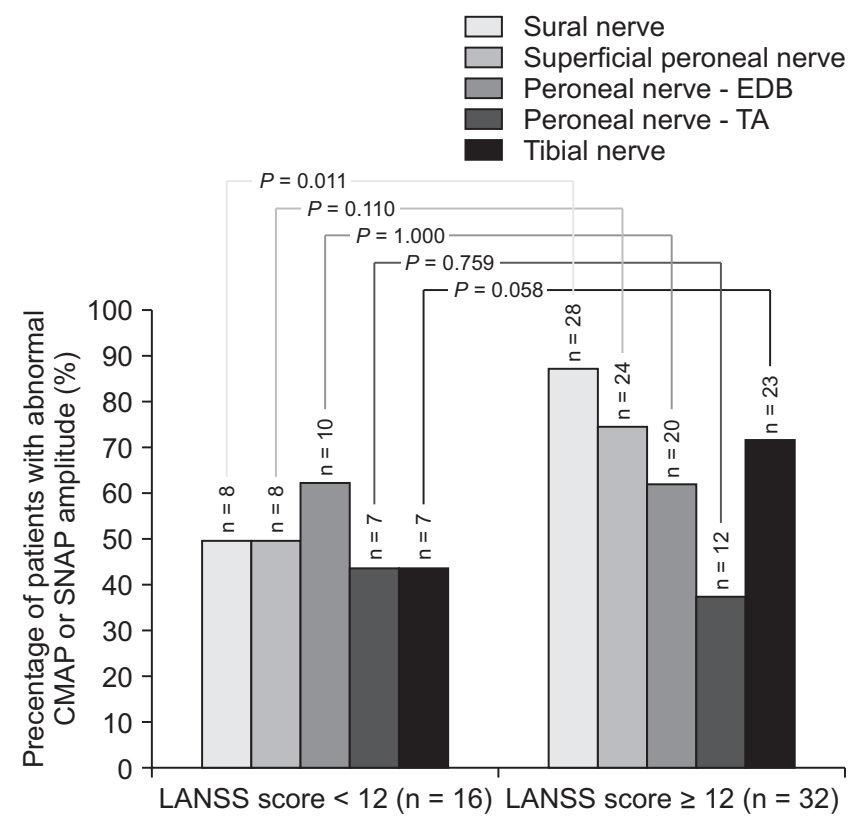

Fig. 2. Compound muscle action potential (CMAP)/Sensory nerve action potential (SNAP) amplitude abnormalities of nerves in patients with total score of Leeds assessment of neuropathic symptoms and signs scale (LANSS-T) score $<12$ and LANSS-T score $\geq 12$. The Pearson's chisquared and Fisher's exact tests were used. A $P$ value of less than 0.05 was considered significant. EDB: extensor digitorum brevis, TA: tibialis anterior.

of 46 patients, and peroneus longus muscle of 45 patients. Active denervation or neurogenic MUAPs were present in the TA muscle of 33 patients ( $69 \%)$, medial gastrocnemius muscle of 32 patients (67\%), peroneus longus muscle of 26 patients (58\%), and short head of the biceps femoris muscle of $31(67 \%)$ patients. There were no patients with electrodiagnostic findings compatible with lumbosacral radiculopathy/plexopathy or polyneuropathy.

The patients' mean LANSS-A, LANSS-B, and LANSS-T
Table 3. Correlation between SNAP/CMAP amplitude grading and LANSS scores

\begin{tabular}{llccc}
\hline \multicolumn{1}{c}{ Nerve } & & LANSS-A & LANSS-B & LANSS-T \\
\hline Sural & $P$ value & $0.004^{*}$ & $0.003^{*}$ & $0.001^{*}$ \\
& $r$ & 0.407 & 0.419 & 0.476 \\
Superficial peroneal & $P$ value & 0.915 & 0.650 & 0.701 \\
& $r$ & 0.016 & 0.067 & 0.057 \\
Peroneal (EDB) & $P$ value & 0.736 & 0.798 & 0.896 \\
& $r$ & -0.050 & 0.038 & -0.019 \\
Peroneal (TA) & $P$ value & 0.147 & 0.665 & 0.221 \\
& $r$ & -0.213 & -0.064 & -0.180 \\
Tibial & $P$ value & $0.004 *$ & $0.039 *$ & $0.004 *$ \\
& $r$ & 0.403 & 0.299 & 0.410 \\
\hline
\end{tabular}

SNAP: sensory nerve action potential, CMAP: compound muscle action potential, LANSS: Leeds assessment of neuropathic symptoms and signs scale, LANSS-A: pain questionnaire part of the LANSS, LANSS-B: sensory testing part of the LANSS, LANSS-T: total score of LANSS, EDB: extensor digitorum brevis, TA: tibialis anterior.

* If $P<0.05$, it was considered statistically significant.

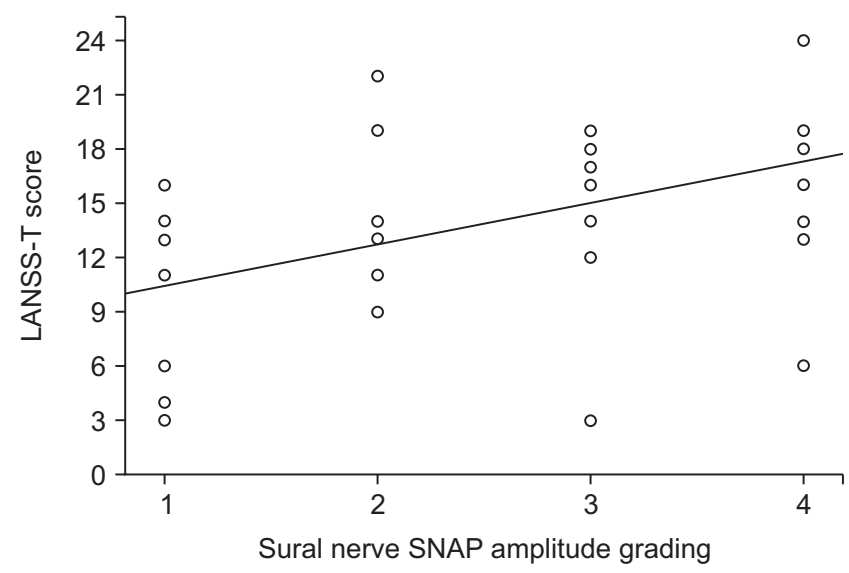

Fig. 3. Correlation between sural sensory nerve action potential (SNAP) amplitude grading and total score of Leeds assessment of neuropathic symptoms and signs scale (LANSS-T) score of the patients.

scores were $9.3 \pm 4.4$ (min-max 0-16), $4.7 \pm 2.5$ (min-max $0-8$ ), and $13.9 \pm 6.0$ (min-max 3-24), respectively. Thirtytwo $(67 \%)$ patients had a LANSS score $\geq 12$. Thirty patients stated that they felt discomfort when something like clothing touched their lower limbs, and 17 patients were diagnosed with allodynia during their neurological examination. The patients were divided into two groups according to their LANSS scores; the number of patients with abnormal CMAP or SNAP in these two groups is shown in Fig. 2. Eight out of the 16 patients with LANSS score $<12$ and 28 out of the 32 patients with LANSS score $\geq 12$ had sural nerve SNAP amplitude abnormality, and the difference between these two groups was significant $(P=0.011$, Fig. 2). The correlation between nerve CMAP/SNAP amplitude grading and LANSS scores is shown in Table 3 . The posi- 


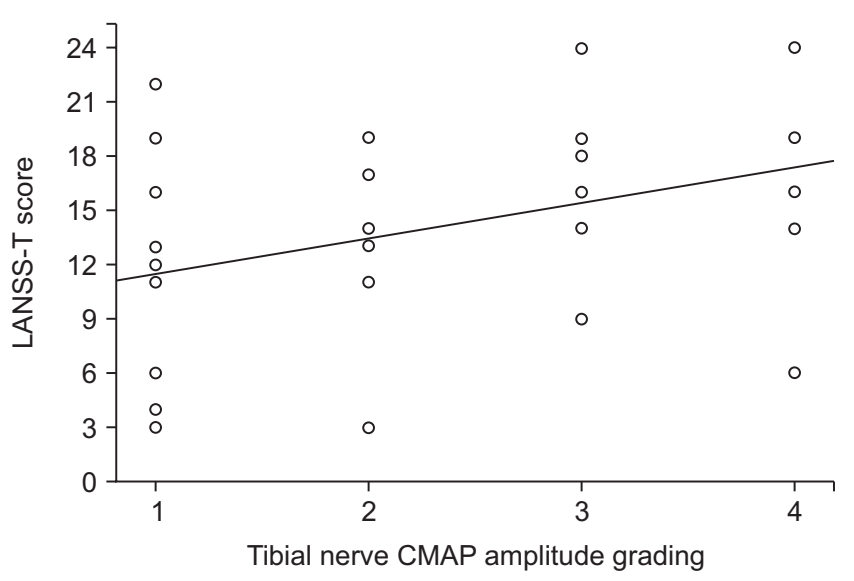

Fig. 4. Correlation between tibial nerve compound muscle action potential (CMAP) amplitude grading and total score of Leeds assessment of neuropathic symptoms and signs scale (LANSS-T) score of the patients.

tive correlation between sural SNAP amplitude grading and LANSS-T scores is shown in Fig. $3(P=0.001, r=0.476)$. Also, Fig. 4 shows the positive correlation between the tibial nerve CMAP amplitude grading and the LANSS-T scale scores $(P=0.004, \mathrm{r}=0.410)$.

\section{DISCUSSION}

Gluteal IM injection is one of the important causes of SN injury. Factors such as low protective tissue, angle of injector, and neurotoxicity of the drug are associated with SNIII $[1,8-10]$. For instance, if the drug is applied directly to the nerve, patient complaints begin immediately; if it is applied very close to the epineurium, the complaints begin a little later. Also, drugs with high neurotoxic effects can injure the nerves even if it they are not administered near them. Similar to previous studies, in most patients, complaints began within seconds of the injection $[1,11,12]$.

It is reported that the peroneal part of the body is more prominently affected in SNIII [1-3]. This may be because the peroneal division is more lateral than the tibial one, or has less protective tissue $[1,12]$. Our findings were consistent with previous research that stated that weakness in the muscles innervated by the peroneal nerve and sensory abnormality in the skin areas supplied by the peroneal nerve were more pronounced. Pain and weakness can mask sensory deficits. With careful sensory examination, most patients may exhibit sensory abnormalities, as in our study. The neurological examinations revealed that there was no muscle weakness in three patients and sensory abnormality in two. In all patients, the presence of needle EMG abnormalities in at least one muscle innervated by the SN or its branches, and the abnormal nerve conduction studies in all patients, except one, indicated the im- portance of electrodiagnostic tests. However, it should be noted that in SNIII, CMAPs or SNAPs of some nerves may be normal. The sural nerve SNAP was abnormal in approximately $78 \%$ of patients in this study, which is consistent with the findings of Yuen et al. [2] that sural SNAP is abnormal in approximately $70 \%$ of patients.

The LANSS-T score was $\geq 12$ in $67 \%$ of the patients. In addition, most patients described discomfort when an object touched their lower extremities. These findings showed that neuropathic pain was present in most of the SNIII patients. The number of sural SNAP amplitude abnormality was significantly higher in those with a LANSS score $\geq 12$ than in those with LANSS score $<12$. In addition, there was a positive correlation between the sural/tibial nerve SNAP/ CMAP amplitude grading and LANSS scores. In one study, injury of the tibial and sural nerves in rats was reported to be more associated with pain than peroneal nerve injury [13]. Although the study was conducted in rats, and the sural nerve was one of the three major branches of the $\mathrm{SN}$ in rats, the findings in that study may support our findings. In that study, the tibial, peroneal, and sural nerves were injured separately or in combination with each other. It showed that rats with tibial and sural nerve injuries had more severe spontaneous pain and mechanical or cold allodynia than those with peroneal nerve injuries. Although Lee et al. [13] stated that the reason for this situation is difficult to explain, they suggest that most of the skin area of the hind paw of rats is supplied by the tibial and sural nerves rather than the peroneal nerve. A large denervated skin area can be innervated by collateral sprouting from the femoral nerve and neuropathic pain may have occurred in this way. When the peripheral nerve is injured, the injured axon can regenerate, or collateral sprouting can occur from an uninjured nerve, thereby ensuring sensory innervation of the denervated skin area $[14,15]$. However, the sensory area supplied by the peroneal nerve in humans is wide. Therefore, the tibial and sural nerves are associated with neuropathic pain in SNIII, which can be explained by other neuropathic pain mechanisms in addition to collateral sprouting.

The pathophysiology of neuropathic pain has not been elucidated yet. Possible mechanisms for neuropathic pain include many conditions such as peripheral or central nervous system sensitization, sprouting, neurogenic inflammation, and increased sodium channels [14-16]. It has been reported that mexiletine or other sodium channel blockers reduced allodynia and hyperalgesia in rats with tibial and peroneal nerve injuries where the sural nerve remained intact [17]. In that study, it was also found that gabapentin reduced mechanical allodynia, but had no effect on mechanical hyperalgesia and cold allodynia, and N-methyl-D-aspartate receptor antagonists did not 
improve allodynia and hyperalgesia [17]. These findings may indicate that one of the important mechanisms may be sodium channel accumulation in SNIII. Accumulation of sodium channels is an important cause of ectopic axonal hyperexcitability which plays a role in pain associated with neuroma $[16,18]$. In a somatosensory evoked potential study of the tibial, peroneal, and sural nerves, the findings suggested that the duration of peripheral receptor activation in the common peroneal nerve stimulation is greater and longer than that of the tibial and sural nerves stimulation [19]. This finding may indicate that the physiological features of the common peroneal nerve differ from tibial and sural nerves and that the responses of these nerves to nerve injury may be different. Characteristics of neuropathic pain may vary in different mononeuropathies due to different etiologies. While pain is an important symptom in carpal tunnel syndrome [20], it may not be an important symptom in peroneal neuropathy at the fibular head due to leg posture [21]. However, the fact that pain is an important symptom of anterior tarsal tunnel syndrome indicates that neuropathic pain is not only due to nervespecific neurophysiological features [22].

It is controversial as to whether there is a correlation between neurophysiological findings and the severity of symptoms [23-26]. Many studies argue that there is no correlation between neurophysiological findings and the severity of symptoms $[23,24,27]$. This situation seems to be the opposite with the findings obtained in our study. It should be noted that some of these studies have been conducted on entrapment mononeuropathies such as carpal tunnel syndrome $[23,27,28]$. Patients whose SN was damaged by the direct or indirect effect of IM were included in this study. Although the correlation found in this study can be explained by the etiology of nerve damage, it is useful to confirm the findings with future studies.

Our results may also be due to changes in the dorsal horn neurons or more proximal structures. It has been reported in animal studies that after SN injury, there are changes in the dorsal root ganglion and spinal cord, or the neurons in the dorsal root ganglion and spinal cord are protected by electrical stimulation [29-31]. Moreover, changes in the dorsal horn neurons were reported as a result of tibial nerve section in rats [29]. Studies on treatment strategies focusing on the tibial and sural nerves and the dorsal root ganglion or spinal cord region associated with these nerves may be interesting in SNIII. One might think that the positive correlation between the tibial nerve CMAP amplitude grading and the LANSS score found in this study is unreasonable, since neuropathic pain originates from the somatosensory nervous system. However, it is known that the somatosensory cortex and motor cortex are in communication with each other. In diseases involving the motor cortex, changes in the somatosensory cortex may also occur, or vice versa $[32,33]$. Nevertheless, considering that the ratio of tibial nerve CMAP amplitude abnormality is not different between SNIII patients with a LANSS score $<12$ and a LANSS score $\geq 12$, we think that the positive correlation found in this study between LANSS scores and tibial nerve CMAP amplitude grading should be confirmed by further studies. In a study of a rat lumbosacral disc herniation model, the persistence of neuropathic pain despite the disappearance of needle EMG abnormalities may indicate that neurophysiological tests such as needle EMG or motor nerve conduction studies, that evaluate motor neuron or axons, will be normal [34].

There were some limitations in this study. First, A delta and $C$ fibers are associated with pain and cannot be examined with routine nerve conduction studies. Therefore, the relationship between routine nerve conduction study findings and LANSS scores found in this study may be confusing. Studies to be conducted with other electrophysiological tests, such as the autonomic function tests in SNIII, will be useful in understanding the pathophysiology of neuropathic pain. Second, the time interval between the complaints of patients and the electrodiagnostic tests ranged from 0.7 to 36 months. Electrodiagnostic findings and LANSS scores may vary depending on the time the electrodiagnostic test is performed. Finally, the sural nerve consists of branches from both the tibial and peroneal nerves, which may also be a limitation. Therefore, degeneration of the tibial and peroneal nerves may have contributed to the sural SNAP amplitude abnormality.

In conclusion, this study showed that there may be a positive correlation between LANSS scores and sural nerve SNAP/tibial nerve CMAP amplitude grading in SNIII. We think that this finding should be confirmed by future studies. In SNIII patients with severely affected sural nerve SNAP, neuropathic pain may be more common.

\section{CONFLICT OF INTEREST}

No potential conflict of interest relevant to this article was reported.

\section{FUNDING}

No funding to declare. 


\section{ORCID}

Halit Fidanc1, https://orcid.org/0000-0001-6573-9090

İlker Öztürk, https://orcid.org/0000-0002-2333-9360

\section{REFERENCES}

1. Jung Kim H, Hyun Park S. Sciatic nerve injection injury. J Int Med Res 2014; 42: 887-97.

2. Yuen EC, So YT, Olney RK. The electrophysiologic features of sciatic neuropathy in 100 patients. Muscle Nerve 1995; 18: 414-20.

3. Tak SR, Dar GN, Halwai MA, Mir MR. Post-injection nerve injuries in Kashmir: a menace overlooked. J Res Med Sci 2008; 13: 244-7.

4. Mishra P, Stringer MD. Sciatic nerve injury from intramuscular injection: a persistent and global problem. Int J Clin Pract 2010; 64: 1573-9.

5. Yucel A, Senocak M, Kocasoy Orhan E, Cimen A, Ertas M. Results of the Leeds assessment of neuropathic symptoms and signs pain scale in Turkey: a validation study. J Pain 2004; 5: 427-32.

6. Fidanci H, Öztürk I, Köylüoğlu AC, Yildiz M, Buturak Ş, Arlier Z. The needle electromyography findings in the neurophysiological classification of ulnar neuropathy at the elbow. Turk J Med Sci 2020; 50: 804-10.

7. Fidanci H, Öztürk İ, Köylüoğlu AC, Yıldız M, Arlıer Z. Bilateral nerve conduction studies must be considered in the diagnosis of sciatic nerve injury due to intramuscular injection. Neurol Sci Neurophysiol 2020; 37: 94-9.

8. Senes FM, Campus R, Becchetti F, Catena N. Sciatic nerve injection palsy in the child: early microsurgical treatment and long-term results. Microsurgery 2009; 29: 443-8.

9. Small SP. Preventing sciatic nerve injury from intramuscular injections: literature review. J Adv Nurs 2004; 47: 287-96.

10. Gentili F, Hudson AR, Hunter D. Clinical and experimental aspects of injection injuries of peripheral nerves. Can J Neurol Sci 1980; 7: 143-51.

11. Yeremeyeva E, Kline DG, Kim DH. Iatrogenic sciatic nerve injuries at buttock and thigh levels: the Louisiana State University experience review. Neurosurgery 2009; 65(4 Suppl): A63-6.

12. Kline DG, Kim D, Midha R, Harsh C, Tiel R. Management and results of sciatic nerve injuries: a 24 -year experience. J Neurosurg 1998; 89: 13-23.

13. Lee BH, Won R, Baik EJ, Lee SH, Moon CH. An animal model of neuropathic pain employing injury to the sciatic nerve branches. Neuroreport 2000; 11: 657-61.

14. Bridges D, Thompson SW, Rice AS. Mechanisms of neuropathic pain. Br J Anaesth 2001; 87: 12-26.

15. Kerstman E, Ahn S, Battu S, Tariq S, Grabois M. Neuropathic pain. Handb Clin Neurol 2013; 110: 175-87.

16. England JD, Happel LT, Kline DG, Gamboni F, Thouron CL, Liu ZP, et al. Sodium channel accumulation in humans with painful neuromas. Neurology 1996; 47: 272-6.

17. Erichsen HK, Blackburn-Munro G. Pharmacological characterisation of the spared nerve injury model of neuropathic pain. Pain 2002; 98: 151-61.

18. England JD, Gamboni F, Ferguson MA, Levinson SR. Sodium channels accumulate at the tips of injured axons. Muscle Nerve 1994; 17: 593-8.

19. Saito T, Yamada T, Hasegawa A, Matsue Y, Emori T, Onishi $\mathrm{H}$, et al. Recovery functions of common peroneal, posterior tibial and sural nerve somatosensory evoked potentials. Electroencephalogr Clin Neurophysiol 1992; 85: 337-44.

20. Oteo-Álvaro Á, Marín MT. Predictive factors of the neuropathic pain in patients with carpal tunnel syndrome and its impact on patient activity. Pain Manag 2018; 8: 455-63.

21. Katirji MB, Wilbourn AJ. Common peroneal mononeuropathy: a clinical and electrophysiologic study of 116 lesions. Neurology 1988; 38: 1723-8.

22. DiDomenico LA, Masternick EB. Anterior tarsal tunnel syndrome. Clin Podiatr Med Surg 2006; 23: 611-20.

23. Truini A, Padua L, Biasiotta A, Caliandro P, Pazzaglia C, Galeotti F, et al. Differential involvement of A-delta and Abeta fibres in neuropathic pain related to carpal tunnel syndrome. Pain 2009; 145: 105-9.

24. Barraza-Sandoval G, Casanova-Mollá J, Valls-Solé J. Neurophysiological assessment of painful neuropathies. Expert Rev Neurother 2012; 12: 1297-309.

25. Oncel C, Bir LS, Sanal E. The relationship between electrodiagnostic severity and Washington Neuropathic Pain Scale in patients with carpal tunnel syndrome. Agri 2009; 21: 146-8.

26. Ortiz-Corredor F, Calambas N, Mendoza-Pulido C, Galeano J, Díaz-Ruíz J, Delgado O. Factor analysis of carpal tunnel syndrome questionnaire in relation to nerve conduction studies. Clin Neurophysiol 2011; 122: 2067-70.

27. Gürsoy AE, Kolukısa M, Yıldız GB, Kocaman G, Celebi A, Koçer A. Relationship between electrodiagnostic severity and neuropathic pain assessed by the LANSS pain scale in carpal tunnel syndrome. Neuropsychiatr Dis Treat 2013; 9: 65-71.

28. Halac G, Topaloglu P, Demir S, Cıkrıkcıoglu MA, Karadeli $\mathrm{HH}$, Ozcan ME, et al. Ulnar nerve entrapment neuropathy at the elbow: relationship between the electrophysiological findings and neuropathic pain. J Phys Ther Sci 2015; 27: 2213-6.

29. Sugimoto T, Ichikawa H, Hijiya H, Mitani S, Nakago T. c-Fos expression by dorsal horn neurons chronically deafferented by peripheral nerve section in response to spared, somatotopically inappropriate nociceptive primary input. Brain Res 1993; 621: 161-6.

30. Hu P, Bembrick AL, Keay KA, McLachlan EM. Immune cell 
involvement in dorsal root ganglia and spinal cord after chronic constriction or transection of the rat sciatic nerve. Brain Behav Immun 2007; 21: 599-616.

31. Pei BA, Zi JH, Wu LS, Zhang CH, Chen YZ. Pulsed electrical stimulation protects neurons in the dorsal root and anterior horn of the spinal cord after peripheral nerve injury. Neural Regen Res 2015; 10: 1650-5.

32. Turco CV, El-Sayes J, Savoie MJ, Fassett HJ, Locke MB, Nelson AJ. Short- and long-latency afferent inhibition; uses, mecha- nisms and influencing factors. Brain Stimul 2018; 11: 59-74.

33. Cengiz B, Fidanci H, Kiyak Keçeli Y, Baltaci H, KuruoĞlu R. Impaired short- and long-latency afferent inhibition in amyotrophic lateral sclerosis. Muscle Nerve 2019; 59: 699-704.

34. Kim SJ, Kim WR, Kim HS, Park HW, Cho YW, Jang SH, et al. Abnormal spontaneous activities on needle electromyography and their relation with pain behavior and nerve fiber pathology in a rat model of lumbar disc herniation. Spine (Phila Pa 1976) 2011; 36: E1562-7. 